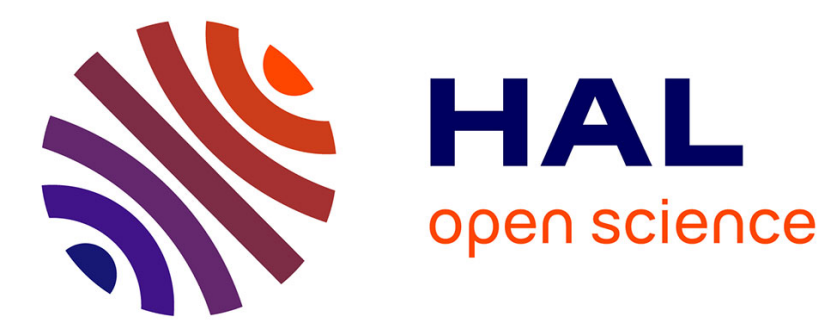

\title{
La gouvernance du foncier agricole périurbain : entre planification urbaine et projets de développement
}

Coline Perrin, Christophe-Toussaint Soulard, Eduardo Chia

\section{To cite this version:}

Coline Perrin, Christophe-Toussaint Soulard, Eduardo Chia. La gouvernance du foncier agricole périurbain: entre planification urbaine et projets de développement. Revue d'économie régionale et urbaine, 2016, 2016 (4), pp.713-736. 10.3917/reru.164.0713 . hal-02633292

\section{HAL Id: hal-02633292 \\ https://hal.inrae.fr/hal-02633292}

Submitted on 27 May 2020

HAL is a multi-disciplinary open access archive for the deposit and dissemination of scientific research documents, whether they are published or not. The documents may come from teaching and research institutions in France or abroad, or from public or private research centers.
L'archive ouverte pluridisciplinaire HAL, est destinée au dépôt et à la diffusion de documents scientifiques de niveau recherche, publiés ou non, émanant des établissements d'enseignement et de recherche français ou étrangers, des laboratoires publics ou privés. 


\section{LA GOUVERNANCE DU FONCIER AGRICOLE PÉRIURBAIN : ENTRE PLANIFICATION URBAINE ET PROJETS DE DÉVELOPPEMENT}

Coline Perrin, Christophe-Toussaint Soulard, Eduardo Chia

\section{Armand Colin | «Revue d’Économie Régionale \& Urbaine »}

2016/4 Octobre | pages 713 à 736

ISSN 0180-7307

ISBN 9782200930653

Article disponible en ligne à l'adresse :

http://www.cairn.info/revue-d-economie-regionale-et-urbaine-2016-4-page-713.htm

\section{Pour citer cet article :}

Coline Perrin et al., « La gouvernance du foncier agricole périurbain : entre planification urbaine et projets de développement », Revue d'Économie Régionale \& Urbaine 2016/4 (Octobre), p. 713-736.

Distribution électronique Cairn.info pour Armand Colin.

(C) Armand Colin. Tous droits réservés pour tous pays.

La reproduction ou représentation de cet article, notamment par photocopie, n'est autorisée que dans les limites des conditions générales d'utilisation du site ou, le cas échéant, des conditions générales de la licence souscrite par votre établissement. Toute autre reproduction ou représentation, en tout ou partie, sous quelque forme et de quelque manière que ce soit, est interdite sauf accord préalable et écrit de l'éditeur, en dehors des cas prévus par la législation en vigueur en France. Il est précisé que son stockage dans une base de données est également interdit. 


\title{
La gouvernance du foncier agricole périurbain : entre planification urbaine et projets de développement
}

\section{Farmland governance in the rural-urban interface : between urban planning and development projects}

\author{
Coline PERRIN \\ INRA UMR 0951 Innovation \\ perrin@supagro.inra.fr \\ Auteur correspondant.

\section{Christophe-Toussaint SOULARD} \\ INRA UMR 0951 Innovation \\ soulard@supagro.inra.fr

\section{Eduardo CHIA} \\ INRA UMR 0951 Innovation \\ chia@supagro.inra.fr
}

Mots-clés : acteur, agriculture périurbaine, aménagement, innovation, Montpellier

Keywords : actor, farmland, France, innovation, urban agriculture

Classification JEL : O2, Q01, Q15, R52 


\section{Résumé}

La planification des usages du sol s'efforce aujourd'hui d'articuler aménagement et développement durable. Les approches par projets ont pris de l'importance par rapport aux approches réglementaires. Notre article analyse comment ces évolutions modifient le statut du foncier agricole dans les projets de développement des communes périurbaines. Pour cela, nous combinons deux approches : l'étude de la planification urbaine et celle du développement agricole local. Les trajectoires de trois communes périurbaines de Montpellier sont reconstituées depuis 1980. La mise en regard des choix d'urbanisme et des projets locaux permet d'identifier trois modes de gouvernance selon la place des agriculteurs et l'importance de la participation. À côté des instruments imposés par l'État, l'utilisation de plus en plus fréquente d'autres instruments, librement mobilisés, relativise le poids de la planification: des concertations et des projets peuvent amener à changer de parti d'aménagement, faisant du foncier agricole non plus un support mais l'objet d'un projet de développement agricole périurbain.

\section{Abstract}

Nowadays, land use planning must balance urbanization and sustainable development. Projects have gained importance over regulatory approaches. This article examines how these changes affect the status of farmland in development projects of peri-urban municipalities. For this, we combine two approaches: we study urban planning and the local agricultural projects. The trajectories of three peri-urban municipalities close to Montpellier, France, are tracked since 1980. This enables us to identify three modes of governance according to the place of farmers and to the importance of participation. Besides the tools imposed by the State, the rise of freely adopted tools minors the weight of planning: consultations and projects can change local planning strategies, considering agricultural land no longer as a support but as the subject of a peri-urban agricultural development project. 


\section{- 1 - \\ Introduction}

Le maintien des terres agricoles face à l'étalement urbain est un enjeu ancien de l'aménagement du territoire (BRYANT et JOHNSTON, 1992). Des années 1970 aux années 1990, l'urbanisme réglementaire a été l'outil principal de contrôle des usages du sol, avec pour objectif majeur l'aménagement d'espaces bâtis. Depuis les années 2000 et la loi SRU (solidarité et renouvellement urbain), la planification des usages du sol s'efforce d'articuler aménagement et développement durable. Les mesures visant à protéger le foncier agricole périurbain se sont multipliées, à la croisée du droit rural, du droit de l'urbanisme et du droit de l'environnement (MARTIN, 2013). À ces approches réglementaires se sont ajoutées une diversité de dispositifs incitant les acteurs à définir eux-mêmes leurs objectifs dans la perspective d'un développement local concerté. Ces constructions font apparaître de nouveaux acteurs, les chargés d'études par exemple, et de nouveaux dispositifs de dialogues, sous forme d'instances de concertation (CHIA, 2013). Aujourd'hui, les collectivités locales en charge de l'aménagement doivent non seulement tenir compte des outils réglementaires, mais aussi faire participer leurs populations à l'élaboration de projets de territoire. Cette évolution vers une gouvernance plus participative modifie-t-elle la prise en compte du foncier agricole dans la planification urbaine? Favorise-t-elle l'agriculture périurbaine?

Les recherches sur le foncier agricole ont déjà abordé ces questions. La prise en compte du foncier agricole dans les documents d'urbanisme a été étudiée dès les années 1970 par des sociologues (MARIÉ et VIARD, 1977) et des économistes (RENARD, 1980). Les économistes ont décrit les mécanismes de la rente foncière d'urbanisation expliquant les comportements de spéculation et de rétention foncière des propriétaires de terres agricoles (JARRIGE et al., 2003). Economistes et géographes ont décrit les conflits d'intérêts qui traversent le monde agricole, entre spéculation foncière et projets de production (CADÈNE, 1990 ; DALIGAUX, 1999), ainsi que les conflits d'usages attribués à la multifonctionnalité des espaces agricoles (DARLY et TORRE, 2008). Ces travaux expliquent le blocage du foncier agricole en périurbain, le coût d'accès aux terres, et les nombreuses convoitises dont leurs usages font l'objet. Ils ont été complétés depuis une dizaine d'années par des recherches s'intéressant plus spécifiquement aux jeux d'acteurs que la question foncière soulève à l'échelle locale entre agriculteurs, propriétaires et acteurs publics en charge des plans d'urbanisme (VIANeY et al., 2006 ; Germain et al., 2006 ; PerRin, 2009 ; Peltier, 2010 ; SERRANO et VIANEY, 2011). Leurs résultats montrent que des compromis formels et informels émaillent la gestion foncière " en acte ", celle-ci se complexifiant au fur et à mesure que la sphère d'acteurs impliqués s'élargit. En effet, à côté des services de l'État, d'autres institutions et des acteurs privés contribuent à la régulation des espaces agricoles périurbains.

Toutefois, la question de la gouvernance du foncier agricole périurbain est encore peu abordée. Quelques travaux situent le foncier agricole dans une réflexion plus globale sur les processus de développement, à travers des publications interdisciplinaires (DuVERNOY et al., 2005). L'ouvrage récent, coordonné par BERTRAND (2013), montre 
à partir des regards d'économistes, géographes, sociologues, gestionnaires et juristes à quel point l'action publique foncière a changé, non seulement par la nécessité d'articuler différents échelles de décision, mais aussi parce que les approches par projets ont pris le pas sur les approches réglementaires (POULOT, 2011 ; 2014). Cependant, ces travaux ne montrent pas comment les acteurs s'emparent du foncier dans la planification urbaine en menant parallèlement des stratégies de développement agricole. On peut alors se demander si la « logique projet » permet l'articulation entre aménagement et développement? Et, si oui, dans quelles conditions cela s'opère-t-il ?

Nous tentons dans cet article de répondre à ces questions en croisant la géographie et les sciences de gestion autour du concept de gouvernance du foncier agricole périurbain. Du point de vue de la géographie, nous abordons ce concept en repérant les changements de statut et d'usages des espaces agricoles, ainsi que la place qu'ils prennent ou pas dans des projets locaux. Du point de vue des sciences de gestion, le foncier est vu comme un domaine d'intervention où différents "instruments " sont mobilisés par les acteurs pour intervenir. Selon HATCHUEL et WeIL (1992), les instruments sont des artefacts sociotechniques combinant un substrat technique, une philosophie gestionnaire et une vision simplifiée des relations organisationnelles ${ }^{1}$. Les instruments sont connus pour le rôle majeur qu'ils jouent dans la conduite des organisations (BERRY, 1983 ; MOISDON, 1997 ; CHIA et al., 2008 ; AGGERI et LABATUT, 2010) et dans la mise en œeuvre des politiques publiques (LASCOUMES et LE GALÈs, 2005). Les travaux de REY-VALETTE et al. (2014) ont confirmé ces résultats pour la gouvernance territoriale en Languedoc-Roussillon : des instruments très divers tels que des Plu ou des SCOT, des chartes de Pays ou des groupes techniques départementaux, mais aussi des projets locaux moins formels, vont cristalliser des rapports noués entre acteurs et, en ce sens, vont constituer d'excellents traceurs de l'action collective. Les sciences de gestion offrent alors des grilles d'analyse du rôle des instruments dans les organisations qui peuvent être appliquées à l'analyse de la gouvernance du foncier ${ }^{2}$.

Notre pari dans cet article est donc de retracer les trajectoires locales de gouvernance au prisme du rôle joué par les instruments dans les dynamiques organisationnelles observées. L'objectif de notre article est de comprendre comment s'articulent planification urbaine et développement agricole local. Il s'agit d'analyser comment s'agencent différents modes d'intervention, celui par le zonage et la réglementation d'une part, et celui par le projet agricole et la mobilisation d'acteurs divers d'autre part. Il s'agit aussi de savoir comment ces modes de gouvernance interagissent sur un même territoire.

Notre hypothèse est que les relations locales entre aménagement et développement se tissent au cours du temps, créant des effets de "dépendance au sentier " (PALIER et BONOLI, 1999) $)^{3}$ et ouvrant aussi des fenêtres d'opportunité pour explorer de nouvelles voies. On peut alors se demander si les initiatives locales qui relient villes et agricultures émergent prioritairement sur des territoires où le foncier agricole a été protégé par des documents d'urbanisme. A contrario, assistons-nous à l'émergence 
de projets agricoles dans des territoires jusque-là peu sensibilisés à la préservation du foncier agricole, et si oui, moyennant quels modes de gouvernance?

Pour éclairer ces questions, nous avons réalisé une étude de terrain dans trois communes de la vallée de l'Hérault situées dans la périphérie ouest de Montpellier. Choisir l'échelle de la commune peut surprendre dans un contexte où les intercommunalités sont devenues des acteurs majeurs de la planification urbaine et de l'intervention foncière agricole à travers la mise en place des SCOT ${ }^{4}$ (JARRIGE et al., 2006 ; CERTU et TERRES EN VILLE, 2008 ; LOUDIYI, 2008). Toutefois, la structuration intercommunale est récente, les SCOT lents à se construire, et l'échelon communal reste encore dans de nombreux espaces périurbains la maille principale de gestion territoriale.

L'analyse de trois trajectoires communales dans le périurbain de Montpellier s'appuie sur le dépouillement des archives d'urbanisme et sur des entretiens, suivant la démarche établie par PERRIN (2009) (annexe méthodologique). L'emploi d'une grille d'analyse élaborée dans le projet PSDR Gouv.Innov ${ }^{5}$ (CHIA et al., 2010) permet de reconstituer des modes de gouvernance caractéristiques de différentes trajectoires de développement territorial.

Nous présenterons tout d'abord les communes étudiées et les critères qui ont guidé leur choix. Puis les trajectoires communales seront retracées afin de révéler différents modes de gouvernance. L'analyse des instruments mobilisés par les acteurs permettra enfin de qualifier le statut du foncier agricole dans les dynamiques locales observées, soit comme support, soit comme objet des actions mises en œuvre. En conclusion nous reviendrons sur les relations entre foncier agricole et gouvernance locale en contexte périurbain.

\section{- 2 -}

\section{Trois archétypes de communes périurbaines}

\subsection{L’hétérogénéité du périurbain en France}

Le périurbain peut être vu comme une mosaïque dont chaque carreau serait une commune composée d'un noyau villageois entouré d'ensembles pavillonnaires plus récents. Dans ces communes, les espaces non bâtis dominent le paysage : l'agriculture et la forêt représentaient, jusqu'à récemment, $80 \%$ de l'espace périurbain des agglomérations françaises tel que défini par l'INSEE (CAVAILHÈS et SCHMITT, 2002). Toutefois, la structure de la population se transforme sous l'effet de l'arrivée de populations urbaines. Les chercheurs parlent d'une double hybridation du périurbain, morphologique par le développement urbain dans un paysage rural, et sociale par la coexistence dans un même espace d'usages différents et parfois antagonistes (BANOS et SABATIER, 2010). Cette réalité a été peu à peu intégrée aux documents d'urbanisme, initialement conçus pour programmer l'extension urbaine, mais prenant de plus en 
plus en compte la combinaison entre usages urbains, agricoles et naturels (PERRIN, 2009 ; MELOT, 2009).

Ce phénomène de périurbanisation connaît des dynamiques différenciées dans l'espace et dans le temps. À partir d'une enquête dans le centre et l'est de la France, CHARMES (2007) a identifié trois types de communes périurbaines. Le type I comprend les petites communes situées sur le front rural de la périurbanisation. Encore très rurales et souvent peuplées de quelques centaines d'habitants, ces communes sont au début d'une forte phase de croissance démographique. Le type II comprend les bourgs centres et les chefs-lieux de canton. Ces communes d'au moins 2000 habitants sont des centres de services et d'équipements pour des communes plus petites situées dans leur orbite. Le type III comprend les communes résidentielles situées dans les couronnes périurbaines anciennes, les plus proches du pôle urbain. L'habitat pavillonnaire prédomine et l'espace communal est majoritairement dédié aux activités résidentielles.

Pour comprendre les liens entre gouvernance du foncier agricole et aménagement urbain, nous avons recherché des communes de la deuxième couronne périurbaine de Montpellier où existaient des initiatives reliant ville et agriculture. En effet, on peut penser que le front de périurbanisation est un espace où les choix de développement, entre urbain et agricole, sont les plus ouverts. La réalisation d'un inventaire des actions dans la zone périurbaine ouest, proche de la Vallée de l'Hérault (PERRIN et al., 2013), a permis d'identifier trois communes où des initiatives reliant ville et agriculture étaient à l'œuvre.

\subsection{Trois communes de la région urbaine de Montpellier}

Les trois communes choisies sont situées à 30-40 km à l'ouest de Montpellier ( $c f$. Carte 1). Elles sont formées de villages ou bourgs entourés d'espaces agricoles.

Montpeyroux, qui est une commune très viticole, se rapprocherait du type I de la typologie de CHARMES. Saint-André-de-Sangonis, qui est un bourg périurbain, correspondrait au type II. La troisième, Villeveyrac, viticole mais de taille plus importante, présente un profil intermédiaire entre les types I et II. Cette lecture est confortée par la typologie de communes réalisée par ABRANTES et al. (2010) établie à l'échelle du Languedoc-Roussillon. En bordure de l'aire urbaine de Montpellier, Villeveyrac et Saint-André-de-Sangonis sont parmi les communes en voie de périurbanisation (forte croissance résidentielle) et Montpeyroux parmi les communes à urbanisation potentielle (densité urbaine faible, mais croissance résidentielle).

Du point de vue démographique, ces communes connaissent toute une croissance qui s'est accentuée fortement dans les années 2000 ( $c f$. Figure 1). Cette croissance a été plus précoce et plus forte dans la commune la plus importante, Saint-Andréde-Sangonis, qui a pratiquement doublé sa population depuis 1968. Villeveyrac connaît un fort dynamisme démographique depuis 10 ans, alors qu'à Montpeyroux, la croissance démographique reste modeste. 


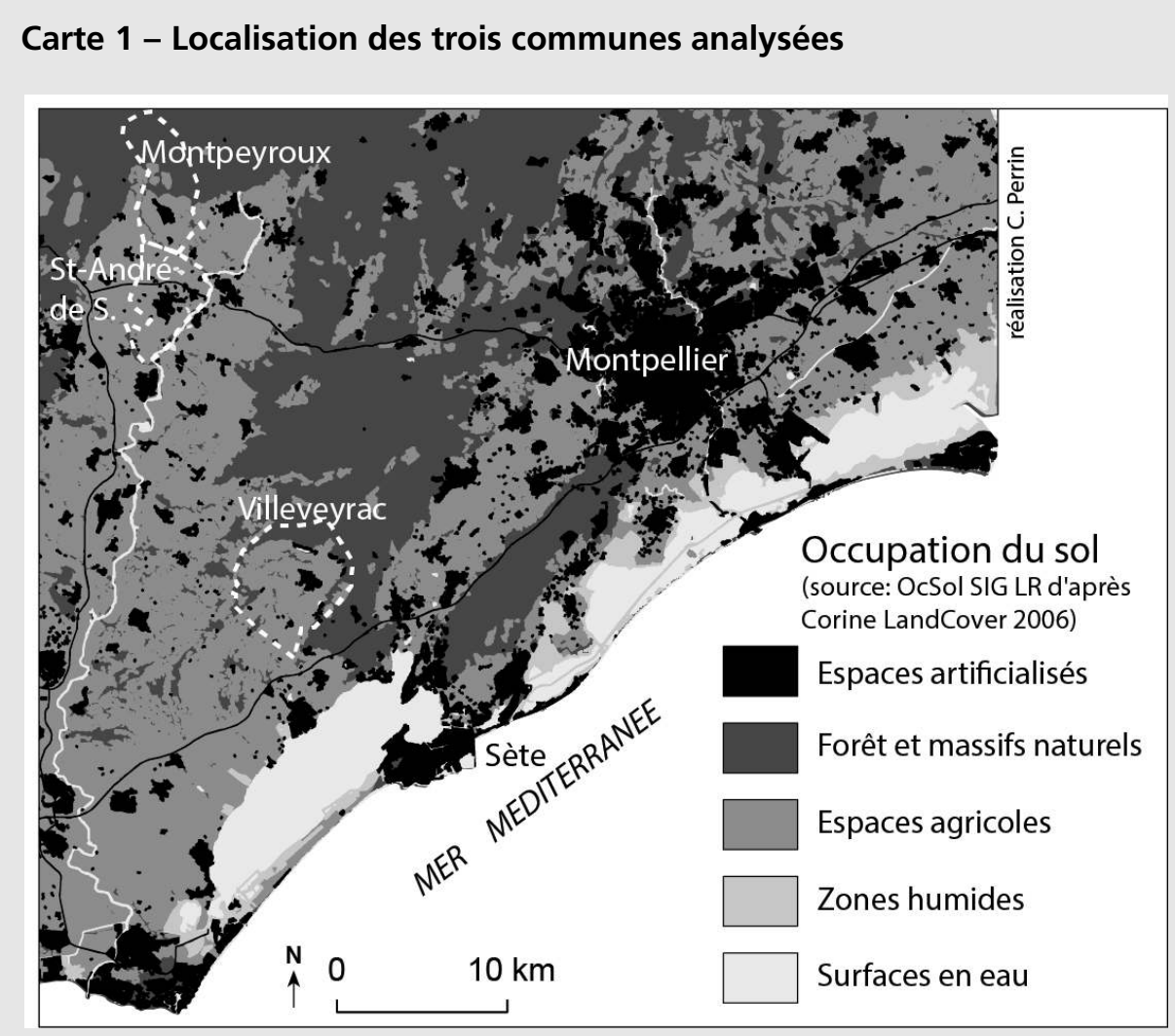

Figure 1 - Évolution démographique des communes étudiées

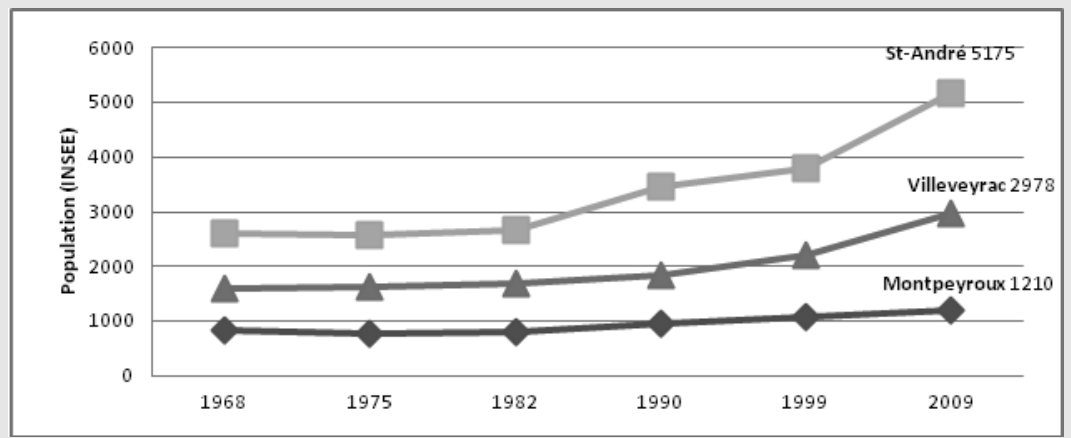

Source : INSEE. 
Du point de vue agricole, la viticulture domine dans les trois communes, malgré des évolutions contrastées ( $c f$. Figure 2). La surface viticole s'est maintenue à Montpeyroux, alors qu'elle connaît un fort recul dans les deux autres communes. Le nombre d'exploitations agricoles s'est réduit dans les trois communes depuis 1979, de $30 \%$ à Montpeyroux, de $60 \%$ à Villeveyac et de $80 \%$ à Saint-André-de-Sangonis. Les autres surfaces agricoles sont également en recul, à l'exception de Montpeyroux où la croissance récente correspond à une reconquête de la garrigue pour l'élevage ovin.

Les trois communes étudiées sont en cours de périurbanisation et à dominante viticole, mais elles présentent des spécificités fortes.

À Montpeyroux (1 200 habitants), la dynamique viticole se maintient. La défense de la ruralité fait consensus, limitant les projets d'extension urbaine. Produisant un vin Aoc de qualité sur des coteaux, la viticulture conserve un poids économique, social et politique important. La commune compte une coopérative dynamique et attire des néo-viticulteurs en cave particulière. Cette logique de valorisation du terroir et du patrimoine est renforcée par l'appartenance de la commune au Grand Site classé de Saint-Guilhem-le-Désert. Située à l'écart des grands axes, Montpeyroux fait figure d'écrin du périurbain montpelliérain.

À l'opposé, Saint-André-de-Sangonis (5 200 habitants), est un ancien bourg rural qui connaît un fort développement résidentiel. Traversée par la nationale et reliée par l'autoroute à Montpellier, cette commune a misé sur le développement résidentiel et économique. Les activités d'extraction de granulats y sont aussi développées. Située sur un terroir de plaine en bordure de l'Hérault, la viticulture de masse n'a pas résisté à la crise. Avec l'urbanisation et l'abandon viticole, la commune a perdu sa vitalité agricole.

Villeveyrac (3 000 habitants) présente un profil médian. La croissance démographique est forte et le vignoble a reculé. Cependant, cette commune a conjugué les deux logiques. Dans les années 1980, la fermeture de la dernière mine de bauxite a incité la commune à miser sur l'agriculture en soutenant le vignoble AOC et l'irrigation. Cet intérêt agricole s'est maintenu sur la période récente, la commune promouvant une agriculture multifonctionnelle de proximité en réponse à la demande des résidents.

Une commune "toute agricole ", une seconde qui a misé sur le " tout urbain ", et une troisième qui conjugue les deux développements, voici les archétypes qui peuvent résumer nos trois cas d'étude. Voyons maintenant leurs trajectoires foncières et les modes de gouvernance territoriale associés. 
Coline Perrin, Christophe-Toussaint SoulARD, Eduardo CHIA

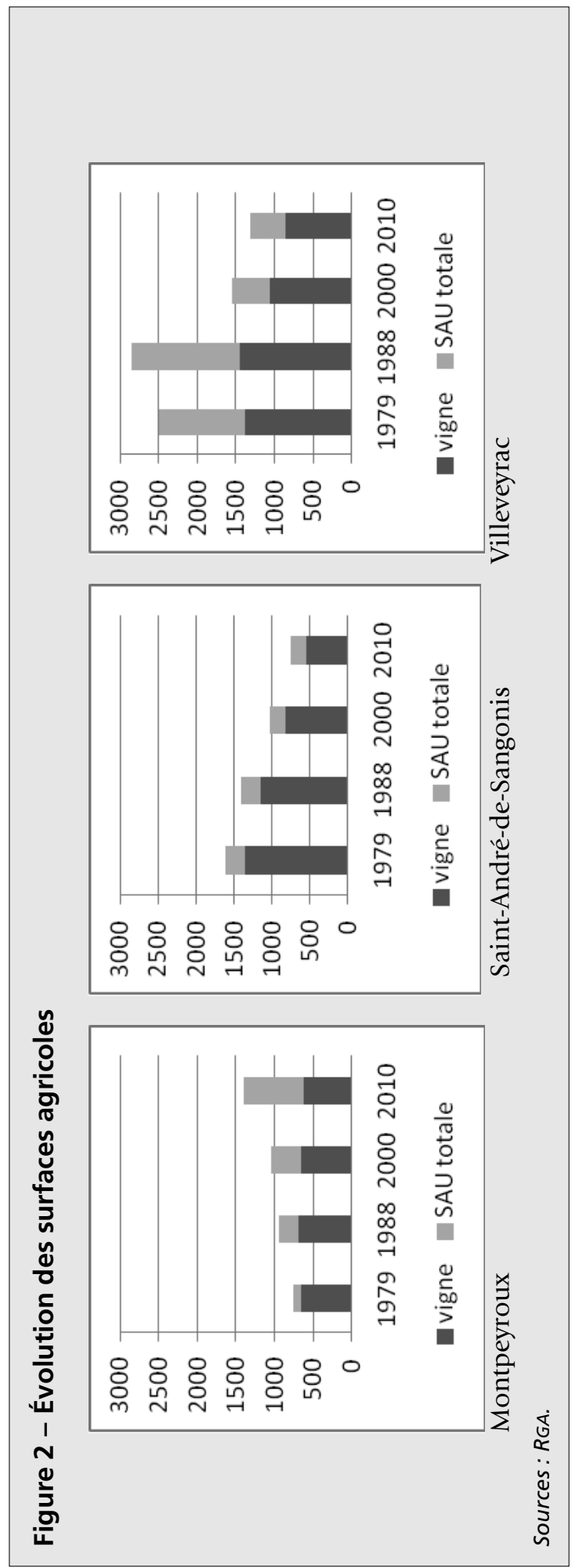




\section{- 3 -}

\section{Planification urbaine et projets agricoles : des relations contrastées}

Les trois communes étudiées ont connu des trajectoires très différentes depuis le début des années 1980.

\subsection{Montpeyroux : protection du foncier et projets agricoles}

À Montpeyroux, le zonage du plan d'occupation des sols (Pos) a été remarquablement stable depuis 1983. Très peu d'espaces agricoles ont été ouverts à l'urbanisation, et ceux qui l'ont été sont situés immédiatement autour des trois hameaux. La modification du Pos en 2005 devait garantir « la pérennité du paysage viticole jusqu'au pied du village ". L'agriculture est présentée comme un enjeu pour l'économie via la production, mais aussi pour le tourisme, car elle contribue à l'identité locale.

Ces choix d'urbanisme protecteurs du foncier agricole ont évité le mitage et la spéculation foncière sur les terres agricoles. Ils tiennent compte de la dynamique agricole locale. La municipalité est présentée par plusieurs acteurs comme à l'écoute des besoins des agriculteurs. Ainsi, en 2002, en même temps qu'elle décide de rendre la zone agricole inconstructible, elle délimite un hameau agricole près du village pour faciliter l'accès au bâti pour les agriculteurs. En 2005, un sous-secteur spécifique a aussi été délimité au sein de la zone naturelle pour permettre l'installation d'un berger sur des terres communales. La municipalité souhaite en effet développer le pastoralisme pour lutter contre les incendies qui ont touché la commune en 1973 et 1983. Dans ce projet, non seulement la municipalité a mis à disposition des terres municipales mais elle a aussi construit une bergerie pour l'éleveur qui s'était installé. La municipalité a par ailleurs soutenu le secteur viticole en défrichant à deux reprises des zones de garrigues municipales et privées, qui ont ensuite été vendues pour permettre l'extension du vignoble et l'installation de jeunes agriculteurs, juste avant la reconnaissance de l'AOc (en 1982) puis dans une période d'embellie du secteur viticole et de forte demande de terres (en 2001).

Développer et conforter l'agriculture est donc un objectif partagé entre la municipalité et le monde agricole local, qui se traduit par des choix d'urbanisme protecteurs du foncier agricole et par des actions municipales en faveur de l'agriculture. Montpeyroux montre ainsi une articulation vertueuse entre planification, besoins des agriculteurs et projets agricoles.

\subsection{Saint-André-de-Sangonis : planification urbaine sans projet agricole}

À Saint-André-de-Sangonis, au contraire, la municipalité a privilégié le développement urbain et les projets agricoles sont rares. Le premier Pos délimite en 1991 de vastes zones d'urbanisation future, pour des lotissements et des zones d'activités. Le 
rapport de présentation indique que "l'économie saint-andréenne vit de la vigne ", il évoque même "la vocation viticole du village ", mais le diagnostic pointe "le vieillissement des chefs d'exploitation et la faible production de qualité ». Aussi, il affirme " la nécessité de trouver des activités de remplacement ou de substitution ".

Ce parti d'aménagement fondé sur l'urbanisation et la diversification de l'économie a probablement accéléré le déclin de l'économie viticole. La cave coopérative était jusque-là dynamique, d'après son ancien président ; elle était engagée dans une démarche qualité fondée sur le réencépagement. Mais après l'approbation du Pos confirmant la volonté d'urbanisation municipale, les friches se développent, les adhérents fuient vers d'autres caves, le président démissionne et la cave fusionne avec celle de la commune voisine en 1998. Selon deux viticulteurs rencontrés, la municipalité se désintéressait déjà de l'agriculture depuis 1977. Contrairement à Montpeyroux, il n'y a donc pas d'alliance entre la municipalité et la cave coopérative, pas de volonté partagée de soutenir l'économie viticole.

Dans les années 2000, cette orientation urbaine se poursuit. Lors de la révision du Pos en 2002, la municipalité se félicite de la forte croissance démographique, renforcée peu après par la construction de la bretelle autoroutière vers Montpellier. Le foncier agricole apparaît avant tout comme une réserve foncière pour la poursuite de l'urbanisation pavillonnaire et le développement de carrières, une activité encouragée par la municipalité parce qu'elle fournit des emplois et une matière première à bas coût pour les constructions. Le plan local d'urbanisme (PLU) approuvé en 2006 est ambigu vis-à-vis de l'agriculture : il constate que " la culture de la vigne reste très présente bien que certains terrains situés sur des zones AOC se soient construits et que les terrains soient souvent envahis de broussaille». (...) «La qualité des productions vinicoles s'étant accrue en Languedoc, il conviendra de soutenir ces initiatives et de promouvoir les compétences des agriculteurs exploitant sur la commune ». Malgré cette affirmation, aucun projet de développement agricole n'a vu le jour. Un hameau agricole a été délimité loin du village sans concertation avec le monde agricole ni même avec le propriétaire. Il n'a donné lieu à aucune construction.

Le changement de municipalité en 2008 s'accompagne de l'élaboration d'un Agenda 21 dans lequel est affichée la volonté de "sauvegarder et développer des emplois agricoles ». Toutefois, plusieurs projets agricoles envisagés - notamment une bourse à la terre pour favoriser la diversification - ont échoué. Le maire nous a parlé d'une "viticulture dans un état catastrophique » qui « ne contribue plus depuis longtemps au développement économique de la commune». Il affirme vouloir protéger les terres agricoles, mais dit aussi devoir faire face à beaucoup de pressions, notamment de la part des agriculteurs-propriétaires anticipant la poursuite de l'urbanisation : "c'est difficile de défendre la vigne contre un vigneron ».

Ainsi, à Saint-André, les choix d'urbanisme ont privilégié l'implantation de lotissements et de zones d'activités et n'ont jamais envisagé positivement l'avenir de l'agriculture. Ce faisant, ils ont accéléré le déclin viticole et n'ont pas permis l'émergence de projets agricoles alternatifs. 


\subsection{Villeveyrac : extension urbaine et projets agricoles}

Villeveyrac présente enfin une trajectoire plus complexe. La municipalité a en effet encouragé simultanément le développement résidentiel et le développement agricole. Le Pos de 1987 ouvre des zones urbanisables importantes autour du bourg et de deux hameaux isolés, sa révision en 1994 délimite de nouvelles zones constructibles à faible densité au sein des espaces agricoles. Dans les années 2000, l'équipe municipale multiplie les documents d'urbanisme (une révision générale, 5 révisions simplifiées et 6 modifications). La cartographie révèle l'extension des zones urbaines sur des parcelles agricoles et la multiplication des sous-secteurs assortis de règlements spécifiques en zones agricoles et naturelles.

Ces choix d'urbanisme ne sont pas incompatibles avec un soutien continu de l'agriculture. Jusqu'en 2000, la municipalité encourage la viticulture. En 1985, la mine ferme au moment où l'AOc est approuvée. Deux projets sont alors lancés : le défrichage du domaine municipal des Capitelles, situé dans le périmètre Aoc, et le lancement d'un réseau d'irrigation pour favoriser la diversification agricole. Ces initiatives expliquent l'augmentation de la SAU communale ( $c f$. Figure 2) et l'installation d'agriculteurs, en particulier les anciens mineurs. Dans les années 1990, la municipalité entreprend aussi une opération de remembrement qui permet l'installation de vingt jeunes agriculteurs. Un chargé de mission à la mairie affirme que l'urbanisme était alors fait « sur mesure pour les agriculteurs » qui souhaitaient pouvoir construire, ce qui explique les "verrues dans le paysage », tandis qu'un viticulteur estime au contraire que leur avis « n'est alors plus systématiquement pris en compte comme avant ».

Le projet des Capitelles évoqué ressemble à celui entrepris à Montpeyroux. Dans les deux cas, il s'agit de faciliter l'accès au foncier des viticulteurs en mobilisant du foncier public, en lien étroit avec la cave coopérative. Toutefois, dans le cas de Villeveyrac, la municipalité reste propriétaire des 100 ha défrichés. L'attribution de baux sur trente ans s'effectue au tirage au sort à une vingtaine d'agriculteurs.

Dans les années 2000, la politique agricole municipale évolue : la municipalité se détourne de la viticulture pour favoriser la diversification agricole et le lien avec les nouveaux résidents. L'agriculture ne représente en effet plus que 4,1\% des actifs du village en 2000, loin des $45 \%$ de 1982. En 2005, la cave coopérative, en difficulté, fusionne avec celle de Pinet. La municipalité lance en 2007 un Agenda 21. Son premier objectif est « la promotion d'une agriculture durable ». Plusieurs projets agri-urbains sont mis en place autour des circuit-courts, de l'éducation et de la diversification des activités agricoles.

Les choix d'urbanisme changent avec cette évolution de la politique agricole. La municipalité devient méfiante vis-à-vis d'agriculteurs parfois " promoteurs ». Le rapport de présentation de 2006 mentionne que les établissements d'élevage sont " trop souvent le déguisement d'implantations résidentielles ». Une zone artisanale agricole est délimitée près du bourg pour concentrer les constructions agricoles et éviter le mitage. Depuis 2011, ce hameau est l'unique zone agricole constructible. Ailleurs, seules les extensions sont autorisées en vue de créer des gîtes ruraux. Cette 
politique nouvelle de préservation des espaces agricoles se poursuit depuis 2012 avec un projet de PAEN ${ }^{6}$.

La trajectoire de Villeveyrac se caractérise ainsi par le passage d'une stratégie de développement agricole couplée au développement résidentiel, à une stratégie de protection du foncier agricole dans le cadre d'une économie plus diversifiée.

Ces trois trajectoires montrent des interactions contrastées entre planification urbaine et projets agricoles. L'urbanisme illustre bien sûr des effets de dépendance au sentier : les choix d'urbanisme actuels ne peuvent être compris sans considération du passé. Une commune préserve d'autant plus facilement ses terres agricoles aujourd'hui qu'elle a fait ce choix depuis les années 1980 (Montpeyroux), et cela favorise les projets agricoles par rapport à une commune qui a considéré le foncier agricole comme de la terre à bâtir dans ses plans d'urbanisme et n'a actuellement aucun projet agricole (Saint-André). Mais la commune de Villeveyrac montre a contrario que la protection stricte du foncier agricole dans les documents d'urbanisme n'est pas une condition nécessaire pour impulser des projets agri-urbains. Elle montre aussi que le lien entre planification et projets peut se faire dans le sens inverse : les projets agricoles peuvent conduire à modifier les documents d'urbanisme, dans ce cas pour mieux protéger les espaces agricoles de l'urbanisation diffuse. Ces trajectoires différentes s'expliquent aussi peut-être par le positionnement de la municipalité en matière de gouvernance.

\section{-4 - \\ Trois modes de gouvernance du foncier agricole}

Notion polysémique (BARON, 2003), la gouvernance peut être étudiée en reconstituant les systèmes d'acteurs au sens de CROZIER et FrIEDBERG (1977), en vue d'analyser en quoi les configurations de ces systèmes favorisent ou non la participation des acteurs " profanes » à l'ensemble du processus. La gouvernance vise alors la construction collective d'objectifs et d'actions en mettant en œuvre des dispositifs (agencement de procédures, mesures, connaissances, savoir-faire et informations) multiples qui reposent sur des apprentissages collectifs et qui participent à des reconfigurations institutionnelles et organisationnelles au sein des territoires (CHIA et al., 2010 ; REY-VALETTE et al., 2014). Suivant cette définition, nous avons considéré l'ensemble des projets inventoriés selon la place occupée par les agriculteurs, les collectivités et les habitants dans le système d'acteurs. Nos résultats mettent en évidence trois modes de gouvernance du foncier agricole.

\subsection{Montpeyroux : une gouvernance bicéphale}

Si la municipalité de Montpeyroux fait le choix dès les années 1980 de protéger et d'encourager la viticulture, c'est non seulement parce qu'il s'agit alors de la principale activité économique, mais aussi parce que le maire de l'époque, d'origine agricole, entretient des relations privilégiées avec la cave coopérative, présidée par son frère. 
Ensemble, ils mettent en place un plan d'aide à l'installation des jeunes viticulteurs. Malgré le changement de maire, cette gouvernance bicéphale se poursuit dans les années 1990. Elle s'institutionnalise en 2000 avec la création d'une commission agricole qui joue à la fois le rôle d'instance de dialogue, d'expertise, de mise en relation avec des acteurs extérieurs (SAFER, Chambre d'agriculture) et de lieu d'expression des logiques agricoles. Cette commission est à l'origine d'apprentissages en matière de gouvernance du foncier (travail collectif, gestion de l'installation).

Cependant, les différentes actions engagées en faveur de la viticulture associent peu ou pas d'autres acteurs de la commune. De leur côté, les chasseurs mènent par exemple des actions d'entretien des parcelles de garrigues avec l'accord du maire mais sans son appui. Ils organisent pour l'école des sorties thématiques sur les plantes aromatiques, la gestion de la chasse, l'entretien du territoire.

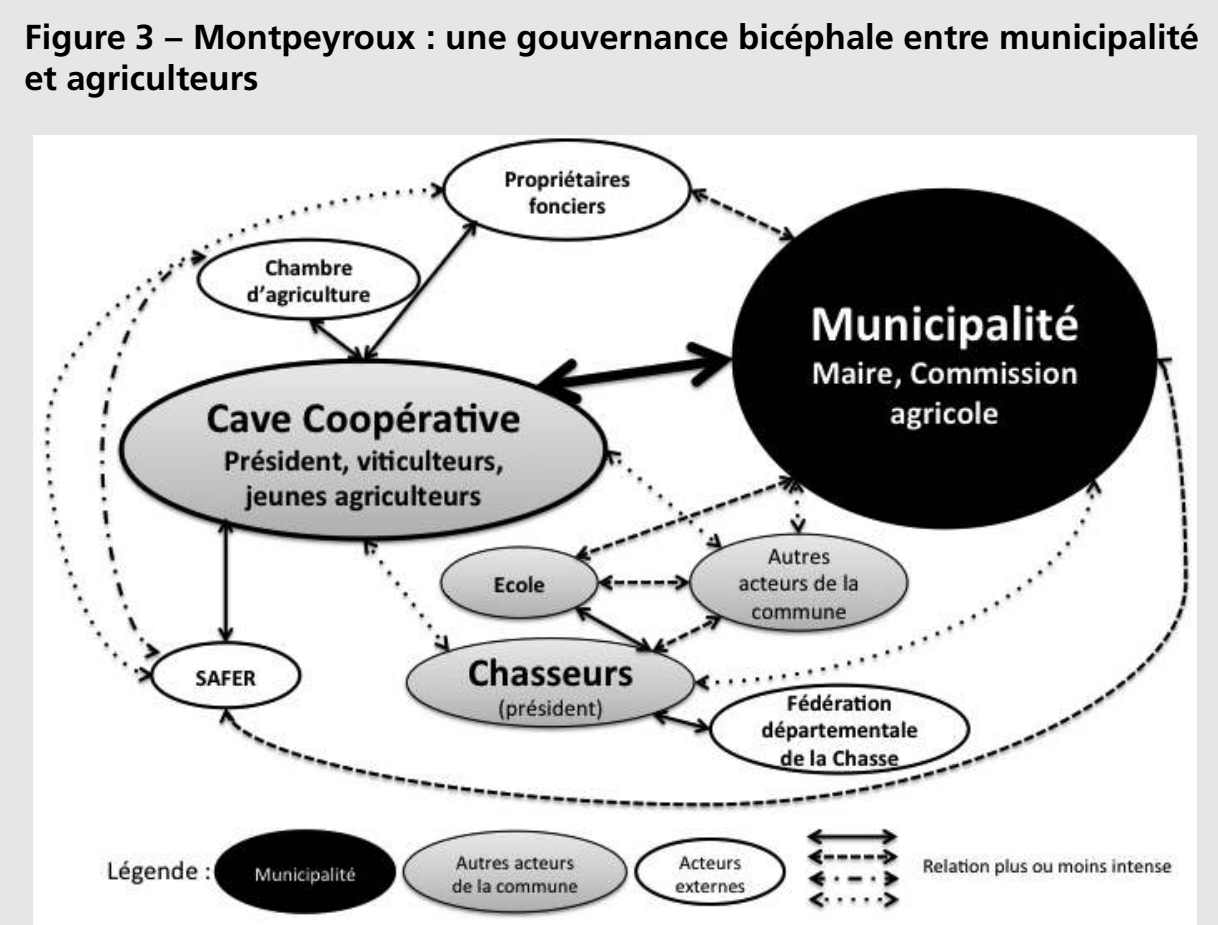

SOUrCe : AUTEURS.

Les chasseurs élargissent ce faisant la gamme de projets agricoles de la commune, replaçant l'agriculture dans le cadre de la protection de l'environnement et du développement durable. 


\subsection{Saint-André-de-Sangonis : une gouvernance monopolisée}

Le système d'acteurs de Saint-André est centré sur la municipalité. L'agriculture n'est pas une priorité : les agriculteurs représentaient $60 \%$ du conseil municipal en 1977, ils ne sont plus que deux conseillers aujourd'hui. Ils ne constituent pas des alliés potentiels pour la municipalité qui a misé sur le développement urbain dès le premier Pos.

Seuls quelques habitants ont participé à la gouvernance territoriale à l'occasion de l'Agenda 21. Des projets créant du lien entre les citadins et l'agriculture ont été menés à bien : un marché de producteurs et un jardin collectif. Mais ils sont portés par des nouveaux résidents (et l'association Passe Muraille) et n'impliquent pas les agriculteurs locaux.

\section{Figure 4 - Saint-André-de-Sangonis : une gouvernance monopolisée par la mairie}

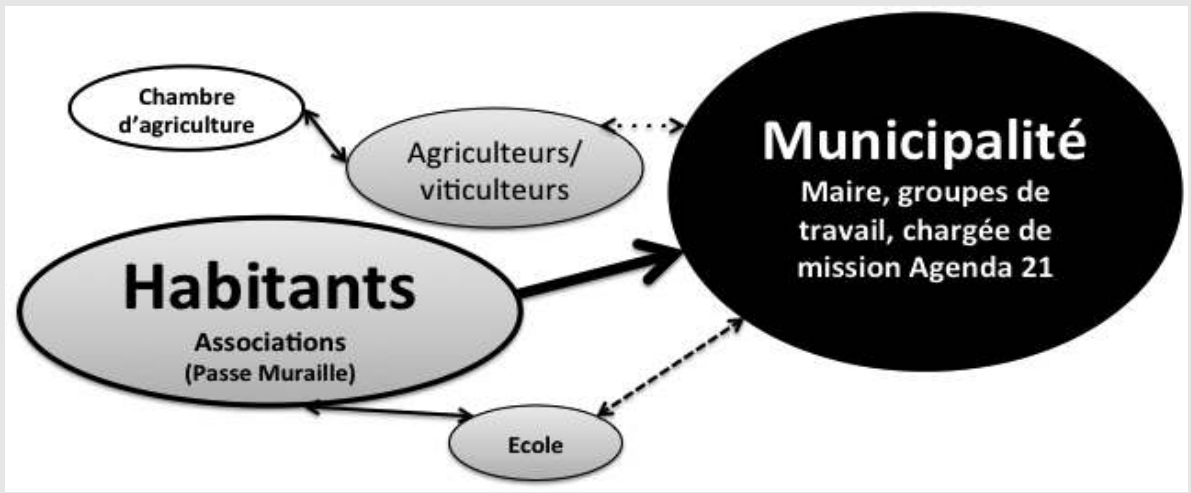

Source : AUTEURS.

L'élaboration d'un Agenda 21 sur un mode participatif était une promesse de campagne des élections municipales de 2008. L'idée venait d'un couple de nouveaux habitants. Sa mise en œuvre a rassemblé divers acteurs locaux dont des agriculteurs. Elle a créé des arènes de discussion sur les visions du développement local. La municipalité a embauché une chargée de mission Agenda 21. Toutefois, le maire s'est progressivement désengagé : il a limité le budget alloué aux projets et n'a pas reconduit la chargée de mission. En l'absence de soutien institutionnel, les autres acteurs initialement impliqués se sont également désengagés. La gouvernance apparaît donc monopolisée par la municipalité.

\subsection{Villeveyrac : une gouvernance plus participative}

À Villeveyrac, le système d'acteurs a évolué, passant d'une gouvernance bicéphale entre la municipalité et les agriculteurs à une gouvernance plus participative. 


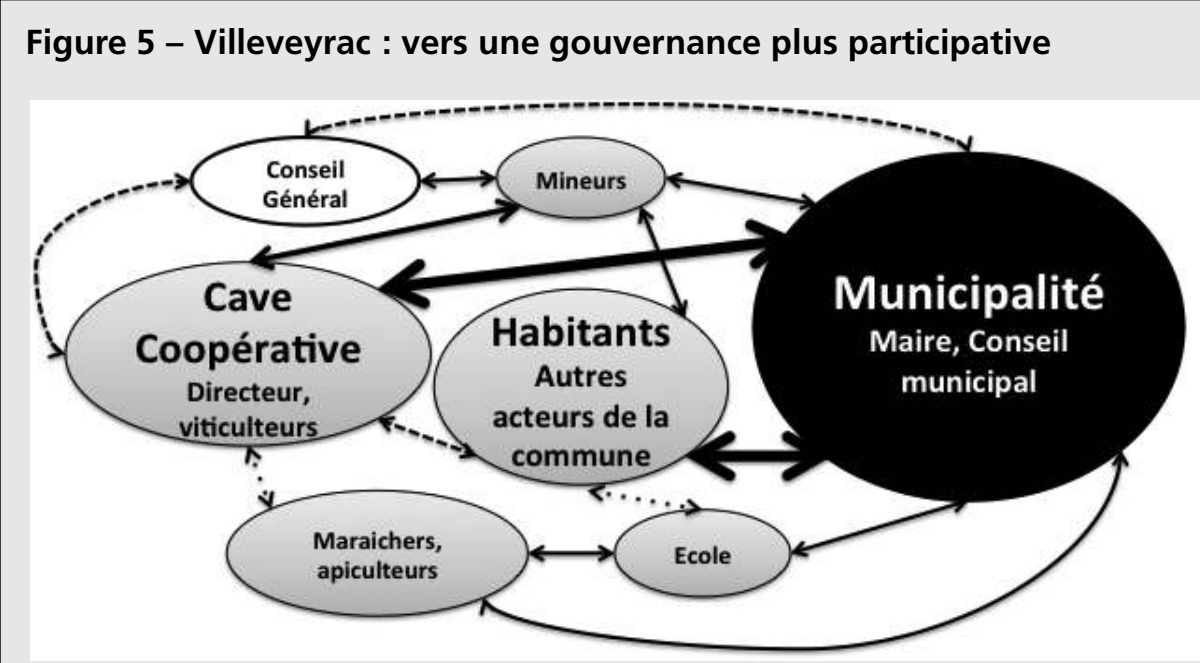

Source : AUTEURS.

Dans les années 1980, les viticulteurs constituent les principaux alliés de la municipalité, comme à Montpeyroux. Lorsque la municipalité met en place l'irrigation et défriche le domaine des Capitelles, de nombreux agriculteurs font partie du conseil municipal. Le maire est un viticulteur et le directeur de la cave coopérative est premier adjoint. Il préside une commission agricole composée d'élus et d'agriculteurs. « Les viticulteurs n'avaient pas besoin d'aller discuter à la municipalité avec les élus puisqu'ils se voyaient tous les jours dans les vignes ou à la cave » (un viticulteur). Le reste de la population est peu sollicité.

À partir de 2007, avec le projet d'Agenda 21, la participation s'élargit à d'autres acteurs : des nouveaux résidents et des agriculteurs non-viticulteurs, notamment plusieurs maraîchers. Les projets mis en place visent plus le développement des circuits courts que la viticulture. Ils ne concernent plus le foncier. Par exemple, sur le domaine des Capitelles loué à des viticulteurs, certaines vignes ont été touchées par une maladie parasitaire et ont été abandonnées. La municipalité refuse toutefois de vendre les terres aux viticulteurs qui souhaitaient pouvoir investir, elle installe en 2009 des ruches sur les parcelles en friches et l'école participe à la plantation de haies et à la récolte de miel. En 2011, le Plu délimite au sein des Capitelles un parc photovoltaïque. Les viticulteurs ne se sentent plus soutenus par la municipalité, contrairement aux éleveurs, maraîchers et arboriculteurs, lesquels sont plus en phase avec la demande urbaine de circuits courts alimentaires de proximité. L'Agenda 21 favorise donc l'établissement d'une gouvernance locale plus participative, même si la municipalité conserve un rôle central.

Des modes de gouvernance territoriale différents émergent ainsi selon la place des différents agriculteurs et des nouveaux résidents dans la gestion des affaires municipales. Dans deux cas, la viticulture est une activité économique à maintenir et à développer. Les caves sont des partenaires privilégiés et la mairie se dote d'une instance spécifique (commission) pour gérer cette interface. À l'opposé, les 
agriculteurs participent peu aux décisions à Saint-André. Les « profanes » que sont les nouveaux résidents sont quant à eux exclus du système de gouvernance bicéphale en place à Montpeyroux et à Villeveyrac jusqu'en 2005. Ils interviennent lors de l'élaboration d'Agenda 21 à Saint-André et Villeveyrac, mais cette opportunité ne conduit à un mode de gouvernance plus participatif, incluant une diversité d'agriculteurs et de nouveaux résidents, que dans un cas, à Villeveyrac. Les modes de gouvernance et leur évolution dépendent donc peut-être aussi des instruments mobilisés.

\section{- 5 - \\ Au cœur de la gouvernance : instruments et projets}

L'articulation entre planification urbaine et développement agricole se fait de manière différente selon la nature des projets et selon que les instruments mobilisés sont imposés par l'État (les SCOT et PLU sont obligatoires) ou librement adoptés, au sens où les acteurs locaux peuvent choisir « librement " de les utiliser ou non (les Agendas 21 locaux par exemple). Tous ces instruments peuvent faire l'objet d'un déplacement, d'une extension, d'un détournement ou d'une adaptation pour atteindre des objectifs différents (AKRICH, 1998) et, en retour, modifier les comportements des acteurs.

\subsection{La gouvernance foncière par l'application d'instruments « imposés »}

Même si les Pos et PLU sont des instruments d'urbanisme imposés par l'État, ils sont aussi des instruments appropriés par les acteurs locaux pour accompagner des stratégies de développement diversifiées. À Montpeyroux, ces plans doivent défendre la viticulture contre la pression de l'urbanisation alors qu'à Saint-André, ils doivent faciliter le développement urbain. Le même instrument peut donc servir localement des objectifs différents. Dans ce processus d'appropriation, les acteurs développent des apprentissages qui permettent la contextualisation de l'instrument (son adaptation à un territoire spécifique), en même temps qu'ils font évoluer les instruments eux-mêmes (en révisant le Pos), ce qui crée de nouvelles incertitudes.

Les Pos et PLU sont les principaux instruments de planification communaux. Ils considèrent le foncier agricole comme un support d'activités, un espace dont la destination d'usage doit être précisée. À travers le zonage et le règlement, ils canalisent le champ des possibles, mais ils ne créent pas du développement. L'articulation avec les projets agricoles ne peut donc être que secondaire, lorsque le parti d'urbanisme décourage la genèse de projets, ou lorsque la concrétisation d'un projet conduit à la révision du Pos, comme dans le cas de la Bergerie communale de Montpeyroux ou de l'aménagement du domaine des Capitelles à Villeveyrac. 


\subsection{La gouvernance foncière par des instruments « libres "}

À côté des instruments d'urbanisme imposés par les pouvoirs publics, dans les trois communes étudiées apparaissent des instruments librement adoptés par les acteurs locaux. Ces instruments, tels que l'Aoc et l'Agenda 21 local, complètent la technologie gestionnaire de l'aménagement territorial (CHIA et al., 2008). Ils donnent parfois naissance à des innovations organisationnelles et à des apprentissages lors de leur contextualisation, et ils permettent de mieux articuler planification et développement.

L'Aoc est un instrument qui sert plusieurs objectifs. À Montpeyroux et Villeveyrac, la reconnaissance de l'Aoc Coteaux du Languedoc en 1985 a dans un premier temps permis d'améliorer la rentabilité des exploitations (prix plus élevés, débouchés plus stables) et de créer un sens commun parmi les adhérents autour d'une viticulture de qualité. Ce faisant, l'instrument AOC a conduit les viticulteurs à modifier leurs objectifs ou valeurs mais aussi leurs pratiques de conduite des vignes, de commercialisation et de coordination avec d'autres acteurs (chasseurs par exemple). Ils ont appris à faire face aux crises viticoles comme à la progression de l'urbanisation. Dans un second temps, la délimitation de l'Aoc a contribué à protéger le foncier destiné à la production, servant souvent de légitimation pour un zonage agricole ou naturel du POS. L'AOc est donc un instrument librement adopté qui articule planification des usages du sol et développement agricole.

L'Agenda 21 local est un autre instrument libre qui a été mobilisé différemment selon les communes. À Saint-André, l'Agenda 21 a permis des apprentissages dans l'élaboration des projets, par la coordination et la négociation. Cependant, le coup d'arrêt donné par le maire (craignant de perdre le contrôle de la dynamique enclenchée) et l'absence de réaction des acteurs locaux ont stoppé la dynamique. Cet exemple montre que, pour que les innovations territoriales se pérennisent, les apprentissages doivent être stabilisés par l'organisation des acteurs, au-delà des seuls instruments. À Villeveyrac, l'Agenda 21 a effectivement permis la participation d'habitants au service du développement local. Il a permis d'intégrer de nouveaux acteurs dans le processus de gouvernance, de soutenir institutionnellement de nouveaux projets, au-delà de ceux prévus lors de son élaboration. Les apprentissages organisationnels sont stabilisés. Cet instrument libre a permis d'instaurer une véritable culture de projet, instaurant une nouvelle gouvernance et modifiant le parti d'aménagement urbain.

\subsection{Vers une gouvernance par les projets ?}

Les projets jouent désormais un rôle central dans la gouvernance du foncier agricole (BERTRAND, 2013). En effet, qu'ils soient initiés par les élus ou par les habitants, les projets permettent aux acteurs de se créer un langage commun, $\mathrm{d}^{\prime}$ apprendre à travailler ensemble, d'explorer le champ des possibles que ce soit pour défricher la garrigue et installer des jeunes agriculteurs ou pour mettre en place un marché de producteurs. Ces projets sont parfois portés par des acteurs périphériques ou minoritaires (bergerie de Montpeyroux, Agenda 21 de Saint-André). Ils permettent donc, mieux que les instruments imposés de planification, la coordination entre 
acteurs (publics et privés) ayant des logiques différentes (TONNEAU et al., 2013). À travers eux, la participation et les apprentissages peuvent se concrétiser.

On peut ainsi affirmer qu'une gouvernance " par les projets ", s'affirme à côté d'une " gouvernance par les cartes " (LASCOUMES, 2007) et par " les instruments " (LASCOUMES et SIMARD, 2011). Pour comprendre les nouveaux modes de gouvernance du foncier agricole périurbain, il nous semble donc indispensable de combiner l'étude de la prise en compte du foncier agricole dans la planification avec celle de l'adoption d'autres instruments libres qui, parce que les acteurs locaux peuvent choisir de les utiliser ou non, favorisent l'élaboration de projets multi-acteurs.

\section{-6 - Conclusion}

Dans cet article, nous avons identifié différentes trajectoires communales de développement agricole en périurbain. Nous avons formulé des hypothèses sur l'évolution du mode de gouvernance du foncier agricole en portant un double regard sur la planification urbaine et sur les projets de développement qui intègrent des questions agricoles. Nos résultats montrent que comprendre ces phénomènes implique de s'intéresser au temps long des pratiques locales (construction du PLu, révisions) et de croiser les apports de la géographie et des sciences de gestion. En effet, le foncier relève d'une construction sociale sur la longue durée, son inertie, liée à la propriété, aux baux, à la réglementation, s'oppose au temps court des processus de gouvernance liés au développement du fonctionnement en " mode projet ».

Pour expliquer les différences de trajectoires communales, nous étions partis de l'idée que certains choix passés structureraient les situations actuelles (path dependency). Mais nos résultats conduisent à nuancer cette proposition. Ainsi, dans le cas de Saint-André-de-Sangonis, la dépendance au sentier est très forte, alors que dans le cas de Montpeyroux, la politique de protection foncière et de soutien à la viticulture a laissé des portes ouvertes à d'autres acteurs, comme les chasseurs et les éleveurs, qui se sont impliqués dans l'entretien des garrigues, et sont devenus des acteurs du développement agricole local. À Villeveyrac, enfin, la concertation et les projets ont conduit à modifier les documents d'urbanisme, et donc à infléchir un parti d'aménagement qui visait l'extension urbaine, pour mieux protéger les espaces agricoles. Ici, le changement d'orientation s'est fait progressivement, le parti d'aménagement s'inversant sans qu'il y ait véritablement de point de rupture.

Peut-on alors soutenir l'idée que le foncier agricole passerait d'un support d'aménagement urbain à un objet du développement agricole dans ces communes périurbaines?

Oui dans certains cas car de nouvelles formes de gouvernance s'affirment. On passe d'un modèle historique de gouvernement mono ou bicéphale (mairie et cave viticole) vers un mode de gouvernance plus ouvert, plus inventif, engageant une plus 
grande diversité d'acteurs dans des projets qui mobilisent le foncier agricole dans des visées de développement local. Mais ce nouveau modèle est encore instable: à Saint-André-de-Sangonis, l'élaboration concertée d'un Agenda 21 n'a pas permis d'infléchir une gouvernance pilotée par la mairie, alors qu'à Villeveyrac, il a été le vecteur d'un changement de gouvernance. On peut alors faire l'hypothèse que nous sommes dans une phase de transition du mode de gouvernance des communes périurbaines.

En somme, le régime dominant des années 1970-2000, fondé sur la gestion municipale de l'urbanisme encadrée par l'État (top down), tendrait à évoluer vers un régime de gouvernance plus participative dans lequel la gestion du foncier agricole deviendrait un levier de développement parmi d'autres. Cette évolution du régime dominant est influencée par les macro-changements du paysage administratif et réglementaire, qui promeut davantage le collaborative planning, mais aussi par des instruments libres, plus susceptibles de répondre à des attentes sociales diversifiées. Les projets agri-urbains locaux (POULOT, 2011) peuvent alors être vus comme autant de niches d'innovations favorisant l'intégration d'acteurs nouveaux qui expérimentent la transition vers une gouvernance foncière plus participative. Ce cadre d'analyse de la transition, mobilisant les catégories conceptuelles de paysage, de régime et de niche (GEELS, 2003), offre une perspective intéressante pour repenser la gouvernance du foncier agricole périurbain.

Face à cette transition en cours, les recherches futures sur la gouvernance du foncier agricole périurbain devraient prendre en compte une nouvelle dimension : il ne s'agit plus seulement de favoriser la protection du foncier agricole (support) mais de faire du foncier agricole (objet) une ressource territoriale pour articuler, dans des projets de territoire, aménagement urbain et développement agricole.

\section{Remerciements}

Cette recherche s'appuie sur le projet PSDR Gouv.Innov financé par l'INRA, l'IRSTEA et la Région Languedoc-Roussillon et sur le travail de terrain réalisé par Solène RAOuL dans le cadre du projet DAUME $n^{\circ}$ ANR-2010-STRA-007-01 financé par l'Agence Nationale de la Recherche. 


\section{Annexe méthodologique}

Les trajectoires d'aménagement urbain et de développement agricole des trois communes ont été reconstruites par RAOUL (2013) à partir de deux sources :

1. Le dépouillement des archives municipales d'urbanisme. Tous les documents d'urbanisme d'échelle communale ont été analysés (cartes communales, Pos, PLu) dans leurs différentes composantes (rapport de présentation, règlement, cartographie), ainsi que quelques comptes rendus de conseils municipaux et des bulletins d'information municipaux. L'élaboration des premiers Pos datant du début des années 1980 dans les trois communes, cette analyse documentaire a permis de retracer la prise en compte de l'agriculture dans la planification locale sur trente ans (1980-2010). Cette méthode s'inspire des travaux de PERRIN (2009).

2. Trente-cinq entretiens semi-directifs. Une première série d'entretiens informatifs a été effectuée auprès des maires et de quelques acteurs locaux, dont des personnes âgées, afin de comprendre le contexte des évolutions communales et de repérer les outils, les projets et les acteurs qui pouvaient créer du lien entre le développement périurbain et l'agriculture. Une seconde série d'entretiens plus compréhensifs a été effectuée auprès d'acteurs impliqués dans les projets agri-urbains préalablement identifiés en s'assurant d'avoir une diversité de statuts (élu, agriculteur, habitant). Ces entretiens ont permis de comprendre l'histoire de chaque projet et les motivations des différents acteurs en s'inspirant des techniques de la chronique et du récit narratif de dispositifs territoriaux (PAOLI et SOULARD, 2003).

La grille d'analyse de la gouvernance issue du projet Gouv.Innov (CHIA et al., 2010) appliquée à ces deux sources de données a permis de reconstruire les systèmes d'acteurs locaux et d'identifier différents modes de gouvernance. 


\section{Références bibliographiques}

Abrantes P, Soulard C-T, Jarrige F, Laurens L (2010) Dynamiques urbaines et mutations des espaces agricoles en Languedoc-Roussillon (France). Cybergeo: European Journal of Geography [en ligne] http://cybergeo.revues.org/22869

AGGERI F, LABATUT J (2010) La gestion au prisme de ses instruments. Une analyse généalogique des approches théoriques fondées sur les instruments de gestion. Finance, Contrôle, Stratégie 3 (13) : 5-37.

AKRICH M (1998) Les utilisateurs, acteurs de l'innovation. La Documentation française, Paris.

BANOS V, SABATIER B (2010) Les espaces périurbains non bâtis en France : entre publicisation " urbaine » et privatisation « rurale ». Articulo-Journal of Urban Research 3 [en ligne] http://articulo.revues.org/1524

BARON C (2003) La gouvernance : débats autour d'un concept polysémique. Droit et Société 54 : 329-351.

BERTRAND N (dir.) (2013) Terres agricoles périurbaines. Une gouvernance foncière en construction. Quae, Paris.

BERRY M (1983) Une technologie invisible? L'impact des instruments de gestion sur les systèmes humains. Cahiers du Centre de Recherche en Gestion de I'Ecole Polytechnique.

BOUSSARD V, MAUGERrI S (2003) Du politique dans les organisations, L'Harmattan, Paris.

BRYANT C-R, JOHNSTON T-R (1992) Agriculture in the citys countryside. University of Toronto Press, Toronto - Buffalo.

CADÈNE P (1990) L'usage des espaces périurbains. Une géographie régionale des conflits. Études Rurales 118-119: 235-267.

CAVAILHÈs J, SCHMItT B (2002) Les mobilités résidentielles entre villes et campagnes. In : PerRIER-CORNET P (dir.) Repenser les campagnes. DATAR, Paris : 35-65.

Certu, Terres en Villes (2008) Prendre en compte l'agriculture et ses espaces dans les SCoT. Éditions du CERTU, Paris.

Charmes E (2007) Carte scolaire et «clubbisation» des petites communes périurbaines. Sociétés Contemporaines 67 (3) : 67-94.

CHIA E (2013) Repenser la gestion foncière au prisme de ses instruments. In : BERTRAND N (dir.) Terres agricoles périurbaines. Une gouvernance foncière en construction. Quae, Paris : 235-247.

Chia E, Mathé S, Rey-Valette H, Michel L, Soulard C, Nougarèdes B, Jarrige F, Clement C, Barbe E, MARTINAD P, MAUREL P, GuIHENEUF P-Y (2010) Comment étudier (analyser) la gouvernance territoriale ? Mise à l'épreuve d'une grille de lecture. Colloque AISRE - ASRDLF, Aoste, Italie, septembre.

ChIA E, TORRE A, ReY-VAletTE H (2008) Conclusion : Vers une « technologie » de la gouvernance territoriale ! Plaidoyer pour un programme de recherche sur les instruments et dispositifs de la gouvernance des territoires. Norois 209 : 167-177.

Chiapello E, Gilbert G (2013) Sociologie des outils de gestion. Introduction à l'analyse sociale de I'instrumentation de gestion. La Découverte, coll. "Grands Repères », Paris.

Crozier M, Friedberg E (1977) L'acteur et le système. Les contraintes de l'action collective. Seuil Point, Paris.

Daligaux J (1999) Urbanisation et société locale en Provence, L'Harmattan, Paris.

DARLY S, TORRE A (2008) Conflits liés aux espaces agricoles et périmètres de gouvernance en Ile-de-France, Geocarrefour 83 (4) : 307-319.

DuVERnOY I, JARrige F, MOUSTIER P, SERRANO J (2005) Une agriculture multifonctionnelle dans le projet urbain : quelle reconnaissance, quelle gouvernance? Cahiers de la Multifonctionnalité 8 : $87-104$.

GEELS F-W (2002) Technological transitions as evolutionary reconfiguration processes: A multi-level perspective and a case-study, Research Policy 31(8-9): 1257-1274.

Germain P, Le Guen R, Thareau B (2006) La re-territorialisation du développement agricole : le cas de I'agriculture périurbaine d'Angers. Revue d'Économie Régionale et Urbaine 2006/3 : 373-392. 
Halpern C, LASCOUmes P, Le Galès P (2014) L'instrumentation de l'action publique. Controverses, résistance, effets. Presses de Sciences Po, Paris.

HATChuEl, A, WeIL B (1992) L'expert et le système: gestion des savoirs et métamorphose des acteurs dans I'entreprise industrielle; suivi de Quatre histoires de systèmes-experts. Economica, Paris.

JARRIGE F, JOUVE A-M, NAPOLEONE C (2003) Et si le capitalisme patrimonial foncier changeait nos paysages quotidiens? Courrier de l'Environnement de I'INRA 49 : 13-28.

JARRIGE F, ThinON P, NOUGAREDES B (2006) La prise en compte de l'agriculture dans les nouveaux projets de territoires urbains. Exemple d'une recherche en partenariat avec la Communauté d'Agglomération de Montpellier. Revue d'Économie Régionale et Urbaine 2006/3 : 393-414.

Lascoumes P, LE GALÈs P (2005) Gouverner par les instruments. Presses de Sciences Po, Paris.

LASCOUMES P, SIMARD L (2011) L'action publique au prisme de ses instruments. Revue Française de Science Politique $61: 5-22$.

LASCOUMES P (2007) Gouverner par les cartes. Genèses 3 (68) : 2-3.

LOUDIYI S (2008) Le SCoT, instrument de gouvernance territoriale ? La conduite locale de la concertation dans le Pays du Grand Clermont. Norois 209 : 37-56.

MARIÉ M, VIARD J (1977) La campagne inventée ou ce qu'il advient des rapports entre les paysans, leurs communautés et l'environnement urbain dans quatre villages d'un pays de moyenne Provence. Actes-Sud, Le Paradou.

MARTIN S (2013) Défendre I'espace agricole : I'accumulation des textes. In : Bertrand N (Coord.) Terres agricoles périurbaines. Une gouvernance foncière en construction. Quae, Paris : 71-80.

MELOT R (2009) De la gestion des espaces au projet de territoire : les enjeux politiques d'un changement de paradigme juridique. L'Année Sociologique 59 (1) : 177-199.

MoisDon J-C (1997) Du mode d'existence des outils de gestion. Seli Arslan. Paris.

Palier B, Bonoli G (1999) Phénomènes de Path dependence et réformes des systèmes de protection sociale. Revue Française de Sciences Politiques 49 (3) : 399-420.

PAOLI J-C, SOULARD C-T (2003) Notes de lecture sur la notion de « Dispositif ». Communication au colloque RIDT INRA-SAD, Dijon.

Peltier C (2010) Agriculture et projet urbain durables en périurbain : la nécessité d'un réel changement de paradigme, VertigO La revue électronique en sciences de l'environnement 10 (2) [en ligne] https://vertigo.revues.org/10119

Perrin C (2009) Construire les campagnes méditerranéennes. Usages, aménagement et valorisations du foncier agricole périurbain en Provence et en Toscane (1950-2010), Thèse de doctorat en géographie, Université d'Aix-Marseille 1 - Facoltà di architettura di Firenze (Italie), 748 p.

Perrin C, Jarrige F, Soulard C T, (2013) L'espace et le temps des liens ville-agriculture : une présentation systémique du cas de Montpellier et sa région. Cahiers Agricultures 22 (6) : 552-558.

POulot M (2011) Des arrangements autour de I'agriculture en périurbain : du lotissement agricole au projet de territoire, VertigO - la revue électronique en sciences de l'environnement 11 (2) [en ligne] http://vertigo.revues.org/11188

Poulot M (2014) Agriculture et acteurs agricoles dans les mailles des territoires de gouvernance urbaine : nouvelle agriculture, nouveaux métiers ? Espaces et Sociétés 158 (3) : 13-30.

RAOUL S (2013) Planification urbaine et développement agricole durable : analyse comparée d'initiatives locales en périphérie urbaine de Montpellier. Master 2 EDTS (MNHN - AgroParisTech) réalisé à I'UMR Innovation, Montpellier.

Renard V (1980) Plans d'urbanisme et justice foncière. Puf, Paris.

Rey-Valette H, Chia E, Mathé S, Michel L, Nougarèdes B, Soulard C-T, Maurel P, Jarrige F, Barbe E, GUIHENEUF P-Y (2014) Comment analyser la gouvernance territoriale. Mise à l'épreuve d'une grille de lecture. Géographie Économie Société 16 : 65-89.

SERRANO J, VIANEY G (2011) Consommation d'espace agricole et relations entre acteurs privés et publics : un management en faveur de l'artificialisation. Norois 221 (4) : 111-124. 
Tonneau J-P, Coudel E, Piraux M, Sabourin E, Valette E, Chia E (2013) Tyrannie des modèles ou souplesse des projets? La question du développement territorial. Colloque international " Circulations et Appropriations des Normes de l'Action locale », Montpellier, 20-23 Mars.

VIANEY G, BACCONNIER-BAYLET S, DUVERNOY I (2006) L'aménagement communal périurbain : maintenir I'agriculture pour préserver quelle ruralité ? Revue d'Économie Régionale et Urbaine 2006/3 : 347 364.

\section{Notes}

1 - Le substrat technique est la dimension concrète de l'instrument, faite de paramètres qui lui permettent de fonctionner. La philosophie gestionnaire représente le but, l'objectif final donné à l'instrument tel que son créateur l'a conçu. Enfin, la vision simplifiée des relations organisationnelles porte sur les représentations que le créateur de l'instrument se fait des rôles de chacun dans l'organisation, et de la manière dont ceux-ci interagissent les uns avec les autres.

2 - Signalons que les sociologues s'intéressent aussi au rôle des instruments dans les relations entre acteurs (BOUSSARD et MAUGERI 2003 ; CHIAPELlO et GILBERT 2013) et les sciences politiques au rôle des instruments de gestion dans la conduite de l'action publique (HALPERN et al., 2014).

3 - Nous empruntons aux travaux sur la dépendance au sentier l'idée que certains choix passés vont structurer, déterminer des choix futurs mais nous n'utilisons pas leur grille de lecture, plutôt centrée sur l'action publique nationale, pour comprendre l'action publique locale.

4 - Schémas de cohérence territoriale

5 - Projet de recherche 2008-2011 du programme PSDR3 cofinancé par l'INRA, l'IRSTEA et la Région Languedoc-Roussillon.

6 - Périmètre de protection des espaces agricoles et naturels périurbains. 